PART 3

TYPE I (CLASSICAL) CEPHEIDS 


\title{
A PRELIMINARY STUdy OF THE CLASSiCAL CEPHEId P-L RELATION IN THE R, I PHOTOMETRIC SYSTEM
}

\author{
J. D. FER NIE \\ David Dunlap Observatory, University of Toronto, Canada
}

(Read by C. M. Coutts)

\begin{abstract}
It is pointed out that observational work on Cepheids in the $R, I$ photometric system offers a number of advantages: the stars are generally brighter at these wavelengths and so can be more easily and accurately observed; effects of binary companions, which are usually earlier type stars, are minimized; reddening is less, can be more easily determined, and is less liable to differing extinction laws than in the UBV system; line-blanketing problems are smaller; the range in $(R-I)_{0}$ is probably less than in $(B-V)_{0}$, and the importance of the colour term in the P-L-C relation thus reduced.

Available $R, I$ data for classical Cepheids are gathered from the literature, and the P-L, P-C, and P-L-C relations investigated. It is found that the preliminary relations

$$
\begin{aligned}
\left\langle M_{R}\right\rangle & =-2.09-2.93 \log P \\
\langle R-I\rangle_{0}= & 0.20+0.12 \log P \\
\left\langle M_{R}\right\rangle= & -2.77-3.34 \log P+3.4\langle R-I\rangle_{0}
\end{aligned}
$$

represent the data to within $\pm 0.13 \mathrm{mag}$. in $\left\langle M_{R}\right\rangle$ and $\pm 0.02 \mathrm{mag}$. in $\langle R-I\rangle_{0}$.

There is some indication that the intrinsic colours of EV Sct, U Sgr, and S Nor are bluer than hitherto believed, conceivably due to circumstellar reddening.
\end{abstract}

\section{Introduction}

Despite the many years of work that have been lavished on the period-luminosity relation of classical Cepheids, the definitive calibration, if such there be, remains elusive. In particular, there are two areas in which uncertainty remains: the intrinsic colours of Cepheids, and the significance of chemical composition effects. It is the purpose of this paper to suggest that both these problems could be reduced, and other advantages obtained, by working in the RI photometric system rather than the UBV system.

With the advent of extended-red photomultipliers of reasonably high quantum efficiency, it is now possible to carry out routine $U B V R I$ photometry of faint stars with a single photometer (Fernie, 1974). Thus there is no reason why $R I$ data for large numbers of Cepheids should not be easily procurable.

The RI photometric system offers the following advantages:

(1) Cepheids, especially faint Cepheids, are generally brighter at these wavelengths, and so can be measured more easily and accurately.

(2) The binary companions of Cepheids, as.would be expected on evolutionary grounds, are generally of earlier spectral type than their primaries. Such companions can often have a significant effect on the $(U-B)$ and $(B-V)$ colours of Cepheids, but the $(R-I)$ colour should be much less affected.

(3) Interstellar reddening effects are less in the RI system than in the UBV system. The reduction, however, is not as great as might at first be supposed, since the standard 
Whitford reddening curve suggests $E_{R-I} \simeq 0.74 E_{B-V}$, and $A_{R} \simeq 3.0 E_{R-I}$. But even a $26 \%$ reduction in reddening is, of course, welcome.

(4) More important than the reduced reddening, perhaps, is the probability that such things as the ratio of total to selective absorption are less likely to vary from region to region in the Galaxy at $R I$ wavelengths than at $U B V$ wavelengths. Figure 1,

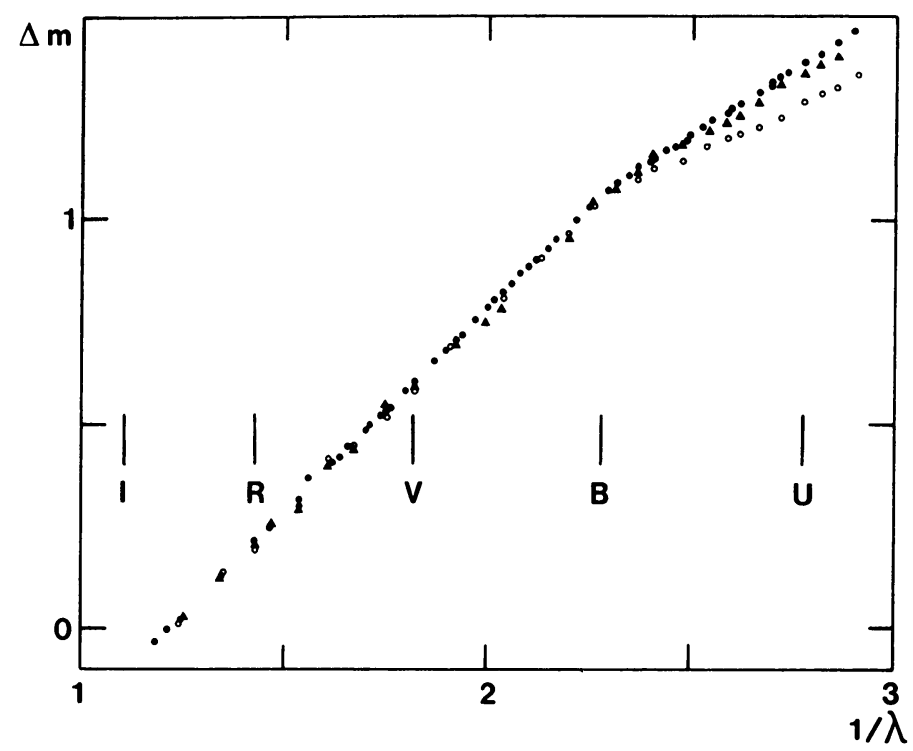

Fig. 1. Variation in interstellar reddening curves. Open circles are for Perseus, closed circles for Cygnus, and triangles for Cassiopeia. (Adapted from Nandy, 1966.)

which is adapted from the work of Nandy (1966), illustrates how the reddening curve can vary from region to region, and how this variation is pronounced at $U$ and probably significant at $B$, but is not so for the longer wavelengths.

(5) If, despite the above, one is prepared to use $(B-V)$ data, then a two-colour plot of $(B-V)$ vs $(R-I)$ enables one to determine colour excesses for Cepheids in much the same way as they are determined for $B$ stars from a $(U-B)$ vs $(B-V)$ plot. The reliability of this procedure and its accurate calibration have yet to be determined, but preliminary results suggest that it offers a significant contribution towards reducing the vexatious problem of Cepheid reddenings.

(6) The question of what variation there is in metallicity among Cepheids, both within the Galaxy and compared to those of external galaxies, remains an open question. If there should be a significant variation in metallicity, however, then problems arising from differing line-blanketing will be much less in $R I$ than in $U B V$.

(7) The amplitude of the light-curve of a given Cepheid is less in $(R-I)$ than in $(B-V)$ by a factor of three of four, and is also much less in $R$ than in $V$. Thus discussions as to whether to use intensity means rather than magnitude means, or to use $\langle R\rangle-\langle I\rangle$ instead of $\langle R-I\rangle$ become academic, since these quantities are essen 
tially equal. In addition, fewer observations are needed to define the light-curves when the latter have much reduced amplitudes.

(8) According to Schmidt (1973), $(R-I)$ is a much better temperature indicator for Cepheids than $(B-V)$, so that presumably theoretical studies would benefit if observational studies were to extend the available $R I$ data.

The use of $R I$ data has, of course, some disadvantages too. For example, there is now a vast body of $U B V$ data available for Cepheids, whereas a switch to $R I$ would entail a great deal of new observing. Perhaps the greatest disadvantage would come in photographic studies of Cepheids in external galaxies, where infrared sensitive plates are considerably less desirable than blue and yellow plates. Nevertheless, the advantages outlined above make it seem worth exploring the period-luminosity relation in terms of $R I$ photometry.

\section{Period-Colour Relation}

As basic data to be used throughout this paper I have restricted myself to those Cepheids which are members of galactic clusters, associations, or binaries, and for which $R I$ photometry is already available. These stars are listed in Table I. The photometric data are to be found in Wisniewski and Johnson (1968), Schmidt (1971),

TABLE I

Basic data

\begin{tabular}{lcccccrc} 
& $\log P$ & $\langle R\rangle$ & $\langle R-I\rangle$ & $E_{R-I}$ & $\langle R-I\rangle_{0}$ & $(m-M)_{0}$ & $M_{R}$ \\
\hline SU Cas & 0.290 & 5.33 & 0.48 & 0.23 & 0.25 & 7.5 & -2.9 \\
EV Sct & 0.490 & 9.08 & 0.76 & 0.50 & 0.26 & 11.3 & -3.7 \\
$\alpha$ UMi & 0.599 & 1.50 & 0.31 & 0.02 & 0.29 & 5.1 & -3.7 \\
U Sgr & 0.828 & 5.80 & 0.75 & 0.48 & 0.27 & 9.0 & -4.6 \\
S Nor & 0.989 & 5.68 & 0.57 & 0.26 & 0.31 & 9.8 & -4.9 \\
$l$ Car & 1.551 & 2.58 & 0.61 & 0.20 & 0.41 & 8.8 & -6.8 \\
RS Pup & 1.617 & 5.82 & 0.84 & 0.44 & 0.40 & 11.2 & -6.7 \\
\hline
\end{tabular}

and Eggen (1971), except those for EV Sct, which come from unpublished observations of my own. All results given here are on Johnson's RI system. The distance moduli shown in Table I are based on a compilation given elsewhere (Fernie, 1967).

Colour excesses have been determined by using $\langle R-I\rangle$ with previously measured $\langle B-V\rangle$ values on a two-colour plot, and taking the slope of the reddening line to be $E_{R-I} / E_{B-V}=0.74$. For the majority of stars the results are in good accord with previously determined colour excesses, but for EV Sct, U Sgr, and S Nor the excesses are significantly larger than those previously found, which were based on the $B$ stars of the three clusters involved. If the previous excesses are adopted, however, these three stars fall well off the period-colour relation shown in Figure 2, so that the present results are at least self-consistent. In addition $\mathrm{S}$ Nor has always appeared surprisingly red for its period, a result usually ascribed to its being close to the redward edge of the instability strip in the H-R diagram. With the excess found here, however, it fits the 


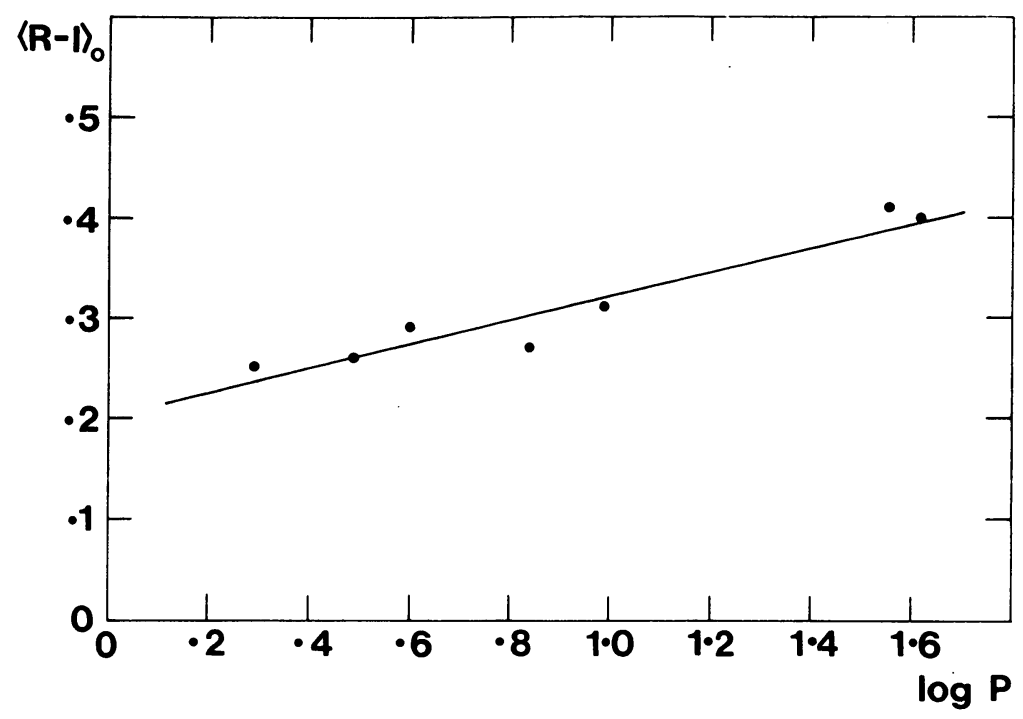

Fig. 2. The period-colour relation for the Cepheids listed in Table I.

mean period- $\langle B-V\rangle$ relation much better too. Conceivably, therefore, there are some Cepheids which suffer additional reddening from circumstellar material, perhaps arising from mass loss. If this effect is confirmed it will mean that the scatter in previously determined period-colour relations is too great, and that the instability strip is narrower than currently believed, mostly in the sense that the redward edge should be moved to the left in the H-R diagram.

Certainly the period-colour relation shown in Figure 2 shows remarkably little scatter. The line in the figure has the equation

$$
\begin{aligned}
\langle R-I\rangle_{0} & =0.20+0.12 \log P \\
& \pm 0.02 \pm 0.02
\end{aligned}
$$

and the mean scatter about it is only $0.02 \mathrm{mag}$. This small scatter may, of course, be fortuitous in view of the small number of stars involved. If not, however, then this very tight correlation implies that the colour term in the period-luminosity-colour relation is essentially redundant. In any case, the change in $\langle R-I\rangle$ with period is much less than is the case for $\langle B-V\rangle$, where the coefficient of $\log P$ is about 0.49 .

\section{Period-Luminosity Relation}

Figure 3 shows the absolute $R$ magnitudes of the Cepheids in Table I plotted as a function of $\log P$. The interstellar absorption corrections to the apparent magnitudes have been taken as $3.0 R_{R-I}$, using the excesses as determined in this study and listed in Table I. Again the correlation is remarkably tight. The line has the equation

$$
\begin{gathered}
\left\langle M_{R}\right\rangle=-2.09-2.93 \log P \\
\pm 0.12 \pm 0.12
\end{gathered}
$$




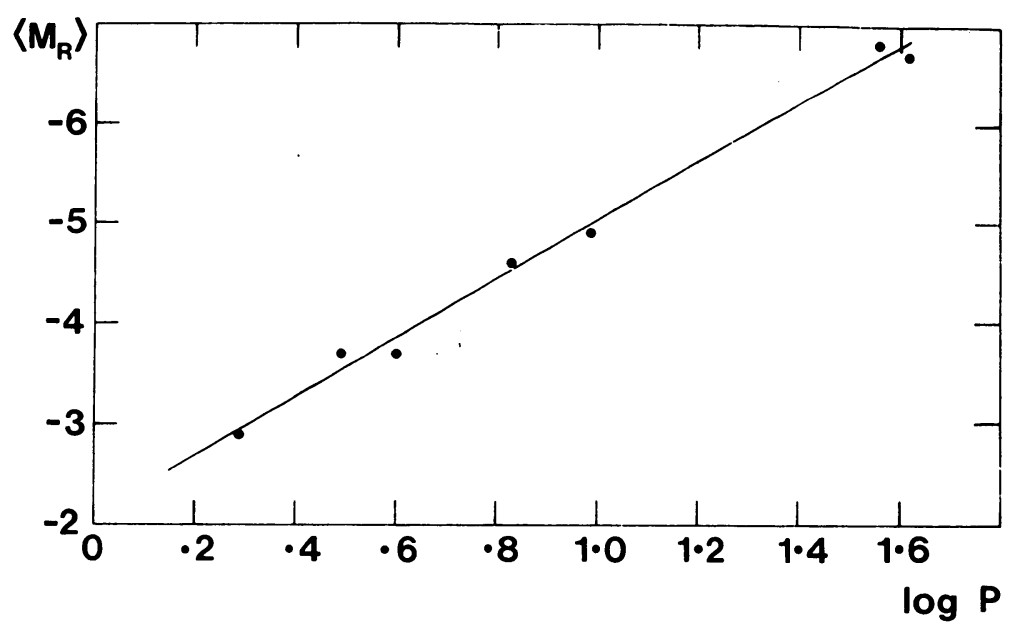

Fig. 3. The period-luminosity relation for the Cepheids listed in Table I.

and the mean scatter is $0.13 \mathrm{mag}$. This again suggests that the colour term in a P-L-C relation is largely unnecessary.

\section{Period-Luminosity-Colour Relation}

Because the scatter in the period-luminosity relation is within the errors of the observationally determined absolute magnitudes of the cluster Cepheids, no hold on the colour term can be obtained from the residuals.

Instead, one may proceed in time-honoured fashion, starting with the $P \sqrt{\varrho}$ relation and successively substituting luminosity and effective temperature for radius, luminosity for mass, a bolometric correction-colour function, and an effective temperaturecolour function to arrive at a P-L-C relation. This proves to be unsatisfactory for several reasons. The masses of Cepheids are currently a matter for dispute, while if one tries to avoid going through $(B-V)$ and to obtain bolometric corrections (here defined as $\left.M_{R-} M_{\text {bol }}\right)$ directly as a function of $(R-I)$, one finds that what little information there is varies greatly depending on its source. The temperature-colour relation, however, is better known (see, for instance, Schmidt, 1973). The available BCtemperature-colour relations suggest that the coefficient of the colour term in the P-L-C is about 3.4. The mass problem could be avoided by a previous procedure (Fernie, 1967), which has as its basis a period-radius relation rather than the $P \sqrt{\varrho}$ relation, but for the present it has seemed adequate to adopt 3.4 for the coefficient of the colour term and then require that the P-L-C relation be compatible with Equations (1) and (2) above. This leads to

$$
\left\langle M_{R}\right\rangle=-2.77-3.34 \log P+3.4\langle R-I\rangle
$$

Applying this to the data of Table I gives a mean residual in $\left\langle M_{R}\right\rangle$ of 0.13 mag., which, as expected, is no improvement over Equation (2), the simple P-L relation. 


\section{Summary}

There are considerable advantages to working in the RI photometric system rather than the UBV system when dealing with classical Cepheids. Available data on Cepheids in clusters, associations and binaries indicate that the period-colour and periodluminosity relations have very little scatter when expressed in this system. There is also a suggestion that some Cepheids may have hitherto had erroneous colour excesses assigned them because of unsuspected circumstellar reddening. In conclusion it is emphasized that the results presented here are preliminary ones only.

\section{Acknowledgement}

This work was supported in part by the National Research Council of Canada.

\section{References}

Eggen, O. J.: 1971, Astrophys. J. 163, 313.

Fernie, J. D.: 1967, Astron. J. 72, 1327.

Fernie, J. D.: 1974, Publ. Astron. Soc. Pacific 86, 837.

Nandy, K.: 1966, Publ. Roy. Obs. Edinburgh 5, 233.

Schmidt, E. G.: 1971, Astrophys. J. 165, 335.

Schmidt, E. G.: 1973, Monthly Notices Roy. Astron. Soc. 163, 67.

Wisniewski, W. Z. and Johnson, H. L.: 1968, Comm. Lunar Planetary Lab., No. 112.

\section{DISCUSSION}

W. Kunkel: There are difficulties with the RI systems for lack of good standards. An instrumental precision of $0 \mathrm{~m} 008$ can be consistently obtained. However, transformation to Johnson's stars [Johnson, H. L., Mitchell, R. I., Iriarte, B., and Wísniewski, W. Z. : 1966, Comm. Lun. Planetary Lab. Tucson 4, 99 (No. 63) frequently leaves a scatter of about $0 m 025$.]

The advantages of the RI system appear sufficiently attractive to merit the establishment of a good set of equatorial standards. Several efforts in this direction have been started, in both, South Africa and at Cerro Tololo.

M. Breger: Did Dr Fernie transform his new P-L-C-relations to $M_{\text {bol }}$ relations? It would be interesting to compare the new $M_{\text {bol }}$ relation with theory and the previous relations involving $(B-V)$.

C. M. Coutts: No.

$D$. H. P. Jones: The difficulty with $R-I$ is that there are three systems available: those of Kron, Johnson and the six-colour. The advantage of Kron's system is that it can be done with an $\mathbf{S} 20$ photomultiplier rather than the $\mathbf{S} 1$ required by Jonson's. S 20 photomultipliers are more sensitive and much easier to handle than S 1's. At the moment Dr Cousins at the Cape of Good Hope is setting up a system of standards in the $E$-regions on a system close to Kron's with an accuracy of a few thousands of a magnitude. 